\title{
Hypersensitivity reactions to drugs in acquired immunodeficiency syndrome
}

\author{
Zvi Ackerman and Micha Levy
}

Clinical Pharmacology Unit, Department of Internal Medicine A, Hadassah University Hospital, Jerusalem 91-120, Israel.

\begin{abstract}
Summary: We describe a patient with AIDS in whom recurrent drug eruption and fever following administration of various unrelated agents developed. Previous drug exposure was uneventful. A possible mechanism for this phenomenon is described.
\end{abstract}

\section{Introduction}

A possible role of viral infection in the induction of adverse drug reactions has been suggested. ${ }^{1} \mathrm{~A}$ well known example is ampicillin rash in infectious mononucleosis. $^{2}$ Acquired immunodeficiency syndrome (AIDS) is caused by infection with retrovirus designated lymphadenopathy associated virus (LAV) or human T-cell lymphotropic virus type III (HTLVIII). ${ }^{3 *}$ Clinically AIDS is characterized by the occurrence of infections with opportunistic organisms, Kaposi's sarcoma and lymphoproliferative neoplasms. Less well known features are increased hypersensitivity to sulphonamides and recrudescence of atopy. ${ }^{4}$

We have recently seen a patient with AIDS who developed drug eruption following the administration of sulphasalazine, ampicillin, carbamazepine and a contrast enhancement medium, urografin. Earlier exposure to penicillins and sulphonamides was uneventful.

\section{Case report}

A 37 year old homosexual was admitted for the evaluation of diarrhoea, weight loss, fever and oropharyngeal candidiasis.

Starting 10 years previously he had recurrent episodes of gonorrhoea, shigellosis and otitis media that were treated with various antimicrobial drugs including penicillins and sulphonamides, without any adverse effects. He had no history of allergic phenomena in the past.

Thirteen months before the present admission the

Correspondence: Z. Ackerman, M.D.

Accepted: 11 August 1986

*Human immunodeficiency virus (HIV)-(Ed). patient underwent evaluation for diarrhoea of 3 months' duration. Proctoscopy revealed slightly hyperaemic mucosa and for the presumptive diagnosis of ulcerative colitis a daily dose of $2 \mathrm{~g}$ sulphasalazine was given. Ten days later a diffuse urticarial rash accompanied with severe pruritus developed. Sulphasalazine was discontinued with gradual fading of the rash. Diarrhoea persisted, he continued to lose weight and developed fluctuating fever up to $39^{\circ} \mathrm{C}$. Oral moniliasis appeared, partially responding to nystatin suspensions. In two of many stool cultures Shigella sonii sensitive to ampicillin was found. Ampicillin $1.5 \mathrm{~g}$ /day was given orally resulting in partial improvement in the frequency and consistency of the stools. Seven days later a diffuse maculopapular rash accompanied with mild pruritus developed. Ampicillin was stopped and the rash gradually faded.

On admission the patient was found to be cachetic and with generalized lymphadenopathy. No skin eruption was seen.

The total lymphocyte count was $1 \times 10^{9} / 1$. Total T lymphocyte (OKT-3) $82 \%$. The proportion of OKT-4 lymphocytes (helper T) 11\% (normal range: $40-50 \%$ ) and OKT-8 (suppressor T) 59\% (normal range: 20 $30 \%$ ) with a ratio of OKT4/OKT8 of 0.2 (normal range 1.5-2.0).

Serological investigation revealed antibodies to HTLV-III (by ELISA) and IgG antibodies in low titre to Epstein-Barr virus and cytomegalovirus (compatible with past exposure to both viruses). Serological tests for brucellosis, rickettsiosis and syphilis were negative as were IgG antibodies for hepatitis A virus and hepatitis B core antigen.

Throat, urine and blood cultures were negative. Chest X-rays were normal. Barium meal revealed slight oedema of the distal ileum. Abdominal computed tomographic (CT) scan with and without 
urografin was normal. A few minutes after completing the abdominal CT scan the patient developed a diffuse urticarial rash without systemic signs of anaphylaxis. The rash resolved spontaneously within a few hours. Two months later a combined sensory-motor peripheral neuropathy developed. Extensive laboratory studies excluded either infectious or malignant aetiology. Carbamazepine $600 \mathrm{mg} /$ day was given with mild relief of the paraesthesia and pain. One week later a diffuse urticarial rash accompanied by fever up to $38^{\circ} \mathrm{C}$ developed. Carbamazepine was stopped with disappearance of the rash and fever. No further allergic phenomena were seen during further 2 months' follow-up.

\section{Discussion}

The patient described herein did not experience earlier any allergic phenomena or drug eruption either to penicillins or sulphonamides although repeatedly exposed to drugs. The propensity to develop allergic reactions occurred following the presentation of

\section{References}

1. Levy, M. The combined effect of viruses and drugs in drug-induced diseases. Med Hypoth 1984, 14: 293-296.

2. Nazareth, B. Ampicillin and mononucleosis. $\mathrm{Br} \mathrm{Med} J$ 1971, 3: 48.

3. Broder, S. \& Gallo, R.C. A pathogenic retrovirus (HTLVIII) linked to AIDS. N Engl J Med 1984, 311: 1292-1296.

4. Seligmann, M., Chess, L., Pahey, J.L. et al. AIDS-An immunological reevaluation. $N$ Engl J Med 1984, 311: $1286-1292$.
AIDS. Hypersensitivity reactions were reported in $78 \%$ of AIDS patients treated with trimethroprimsulphamethoxazole for Pneumocystitis carinii. ${ }^{5}$ Rash was the most common reaction. Other manifestations were fever, leukopaenia, thrombocytopaenia and hepatitis. ${ }^{5}$ Susceptibility to react adversely to ampicillin or other penicillins, to carbamazepine and to urografin has not yet been described in AIDS patients. The cause for the increased rate of hypersensitivity reactions in AIDS patients is poorly understood although defects of both T and B cells may be operative. It is possible that the non-specific allergic reaction observed in our patient (rash and fever) following many drugs was a manifestation of the increased number of polyclonally activated B cells as found in patients with AIDS. ${ }^{6}$ These abnormal cells are defectively activated and secrete immunoglobulins. This leads to the formation of circulating immune complexes and auto-immune phenomena. ${ }^{4}$

Increased hypersensitivity reactions to drugs in AIDS may provide another example of the combined effects of viruses and drugs in the aetiology of druginduced illness.

5. Gordin, F.M., Simon, G.L., Wofsy, C.B. \& Mills, Adverse reactions to trimethoprim-sulphamethoxazole if patients with AIDS. Ann Intern Med 1984, 100: 495-499훙

6. Bowen, D.L., Lane, H.C. \& Fauci, A.S. Immunopatho= genesis of the acquired immunodeficiency syndrome. Ann Intern Med 1985, 103: 704-709. 